Neuromythology

\title{
Romberg's test no longer stands up
}

Martin R. Turner

Nuffield Department of Clinical Neurosciences, University of Oxford, UK

Correspondence: Professor Martin Turner

$$
\begin{aligned}
& \text { West Wing Level } 6 \\
& \text { John Radcliffe Hospital } \\
& \text { Oxford } \\
& \text { OX3 9DU, UK } \\
& \text { +44(0)1865 223380 } \\
& \text { martin.turner@,ndcn.ox.ac.uk }
\end{aligned}
$$

Counts: Body - 789, Refs - 10 


\section{Preface to 'Neuromythology'}

A stereotype of neurologists is their focus on the pedantry and pomposity of the diagnostic chase. Neurologists do, perhaps uniquely, recognise the primacy of a history well taken. The physical examination of the nervous system is a highly prized skill among medical students, but typically becomes radically honed in specialist training and beyond; extremely so for some (1). As a trainee, the author recalls a teaching session where two senior consultants offered to demonstrate “The Definitive Neurological Examination”. Forty-five minutes in, argument was raging between them over the optimal method to test olfactory function. The passion of specialists was obvious, who will inevitably identify aspects of history-taking, examination, or investigation that they feel strongly enough about to reject any dogma taught as medical students. Few consultant neurologists respect the medical student's anatomical division about an imaginary line at the neck, which is testament to a wider need to fully reappraise what is taught to undergraduates (2). That the student bemoans neurologist $\mathrm{X}$ telling them to test it this way but neurologist $\mathrm{Y}$ another way, is a (healthy) sign that there is still a great art to the clinical encounter as a whole. Long live intuitive neurology (3)!

The idea for an irreverent series of short opinion pieces on aspects of neurological examination was inspired in part by a memorable article in this journal (4). Neuropoppycockology appeared under a section entitled 'Neurological rant' which disappointingly did not become a regular feature. Tempting as it was to resurrect this title, it was agreed instead that 'Neuromythology', acknowledging the collected writings of William Landau (5), might serve the aim of providing a column to provoke thought and online debate about what neurologists do and, hopefully, what they also teach. The first of these calls into question the on-going value of Romberg's test. 


\section{Romberg's test}

The process of standing unsupported with eyes closed and feet together for thirty seconds, relies on several integrated networks of the nervous system, including the vestibular apparatus, cerebellum, dorsal columns, trunk and leg muscle tone. The central assertion is that this is neither highly sensitive nor specific for its original purported purpose. In addition to primum non nocere, in this litigious era is it sensible to even consider a test where the positive result is the patient falling to the floor? They do not fall of course. Rather, they may sway a little this way, then a little that way, until clinician or patient loses either their nerve or their interest.

Like several medical eponyms, the test ascribed to Moritz Romberg (1795-1873, medical students nota bene there is no ' $\mathrm{h}$ ' in Romberg), may have been described by others first, namely the German Bernardus Brach, or Englishman Marshall Hall $(6,7)$. The test appeared in the second edition of Romberg's textbook Lehrbuch der Nervenkrankheiten des Menschen in 1853, where he stated it to be pathognomonic of tabes dorsalis, which he termed a "neurosis of motility" (8). It was Gowers however, many years later, who made the link to the dorsal column lesion. As Keppel-Hesselink and Koehler's chapter also notes, Romberg's test long pre-dated any formal neurological examination, including any understanding of reflexes or concepts of ataxia. It also broke new ground in requiring an instruction to be given to the patient (8).

What then has made Romberg's test so peculiarly memorable, indeed so that it has become something of a common denominator in primary care, applied across the full range of neurological symptoms (MRT - personal observation)? With increasingly time-pressured consultations there is a suspicion that Romberg's test has become, for some, something of a screening procedure. If so, it is argued that observation of the patient getting up and walking from the waiting room (or struggling to do so) serves this function well (9). Furthermore, the 
patient with significant sensory ataxia will likely reveal clues to their reliance on vision in their history, for example sitting down before daring to remove a sweater, or ensuring that they are holding on to something when washing their face (indeed they may have abandoned standing in the shower). Furthermore, a faster, more specific test of proprioception can then be performed at the great toe, with the patient safely lying or sitting.

Reminders of more obscure eponymous neurological signs certainly enrich clinical culture (10), and perhaps it is simply that more memorable surnames facilitate their persistence. Conversely, it is disappointing that nameless manoeuvres with important diagnostic value, such as testing neck muscle strength, rarely survive into the clinical practice of the non-neurologist. Romberg's test is appealingly simple to perform, but it lacks essential specificity. Given that it risks physical injury, and is redundant through testing proprioceptive loss at the toe, it is suggested that the modern discerning clinician will omit. 


\section{References}

1. Hawkes CH. I've stopped examining patients! Practical neurology. 2009;9(4):192-4.

2. Nicholl DJ, Appleton JP. Clinical neurology: why this still matters in the 21 st century. Journal of neurology, neurosurgery, and psychiatry. 2015;86(2):229-33.

3. Donaghy M. Intuitive neurology. Brain : a journal of neurology. 2015.

4. Hawkes CH. Neuropoppycockology. Practical neurology. 2009;9(6):347-52.

5. Landau WM. Clinical Neuromythology: Futura; 2001.

6. Lanska DJ, Goetz CG. Romberg's sign: development, adoption, and adaptation in the 19th century. Neurology. 2000;55(8):1201-6.

7. Pearce JM. Romberg and his sign. European neurology. 2005;53(4):210-3.

8. Keppel-Hesselink JM, Koehler PJ. Romberg's sign. In: Koehler PJ, Bruyn GW, Pearce JM, editors. Neurological Eponyms: Oxford University Press; 2000. p. 166-71.

9. Allen C, Scolding N, Mumford C, Smith P, Fuller G. How I start a new patient consultation. Practical neurology. 2013;13(4):254-7.

10. Hilton-Jones D. Beevor's Sign. Practical neurology. 2004;4(3):176-7. 